EESTI NSV TEADUSTE AKADEEMIA TOIMETISED. 29. KOIDE FOOSIKA * MATEMAATIKA. 1980,. NR. 2

ИЗВЕСТИЯ АКАДЕМИИ НАУК ЭСТОНСКОИ ССР. ТОМ 29 ФИЗИКА * МАТЕМАТИКА. 1980, № 2

удК $531.31+531.38$

H. $\mathrm{KEHC}$

\title{
О ДИНАМИКЕ СИСТЕМ ЛАГРАНЖА С ЛИНЕИНЫМ ПО ГАМИЛЬТОНИАНУ И ИМПУЛЬСАМ АГРЕГИРУЮЩИМ ИНВАРИАНТОМ
}

(Представил Н. Алумяэ)

Найдены условия существования инварианта - линейной комбинации $p, H$. Неавтономная система преобразована в автономную, допускающую понижение порядка на две единицы. Предложена модификация принципа наименьшего действия и рассмотрена устойчивость стационарных движений. Получены агрегирующие инварианты и условия устойчивости равновеснй некоторых гамильтоновых систем.

1. Рассмотрим неавтономную динамическую систему $S$ Лагранжа

$$
\left(p_{i}{ }_{i}+\partial H_{1} / \partial q_{i}-Q_{i}\right) \delta q_{i}=0 \quad\left(p_{i}=\partial L_{1} / \partial q_{i} ; q_{j}=\partial H_{1} / \partial p_{j}, i, j=\overline{1, n}\right),
$$

$H_{1}(t, q, p)=p_{j} q_{j}-L_{1}, \quad\left|\partial^{2} L_{1} / \partial q_{i} \partial q_{j}\right| \neq 0, \quad u_{i} v_{i}=\sum_{i=1}^{n} u_{i} v_{i}=u \cdot v, \quad f=d f / d t$, подчиненную идеальным связям, допускающим виртуальное перемещение:

$$
\delta^{0} q=\varepsilon \partial G_{1} / \partial p \quad\left(G_{1}=v_{0} H_{1}+v_{i} p_{i} ; v_{0}, v_{i} \in C_{1}(t), v_{0} \neq 0\right) .
$$

С учетом (1.1), (1.2) функция $G_{1}[t, q(t), p(t)]$ удовлетворяет уравнению

$$
\begin{gathered}
G_{1}=D\left(H_{1}\right)+W(Q)(D(f)= \\
\left.=v_{0} \frac{\partial f}{\partial t}-v_{i} \frac{\partial f}{\partial q_{i}}+v_{0} f+v_{i} p_{i}, W(Q)=\frac{\partial G_{1}}{\partial p} \cdot Q\right) .
\end{gathered}
$$

При $W(Q) \equiv 0$ функция $G_{1}$ будет инвариантом системы (1.1), (1.2) тогда и только тогда, когда $H_{1}$ удовлетворяет уравнению $D\left(H_{1}\right)=0$ на $E^{2 n+1}$. Для этого необходимо и достаточно, чтобы $G_{1}, H_{1}, L_{1}$ в переменных $t, x, p$ были функциями вида

$$
\begin{aligned}
& G_{1}(t, q, p)=G(x, p), H_{1}(t, q, p)=H(t, x, p)=v_{0}^{-1}\left[G(x, p)-v_{i} p_{i}\right](i=\overline{1, n}), \\
& L_{1}(t, q, q)=v_{0}^{-1} L\left(x, v_{0} x\right), x=q+u, u=\int_{0}^{t} v_{0}^{-1} v d \tau\left(f(t, x, p)=f_{1}(t, x-u, p)\right) .
\end{aligned}
$$

Для натуральной системы $S^{+}$, обладающей инвариантом $G_{1}$, из (1.4) имеем 


$$
\begin{gathered}
G(x, p)=1 / 2 b_{i j}(x) p_{i} p_{j}+b_{i}(x) p_{i}+b_{0}(x), \\
L_{1}=1 / 2 v_{0} a_{i j}(x) x_{i} x_{j}+a_{i}(x) x_{i}+v_{0}^{-1} a_{0}(x), \\
\partial H_{1} / \partial t \neq 0 \quad \text { при } v_{i} \partial H_{1} / \partial q_{i} \neq v_{0} H_{1}+v_{i} p_{i} .
\end{gathered}
$$

3 ам ечание 1. Приведем достаточные условия существования у $2(n+m)$-мерной идеальной системы $S^{\prime \prime}$ подсистемы $S^{+}$, удовлетворяющей $(1.1),(1.2),(1.4)$ при $W(Q) \equiv 0$. Рассмотрим систему $S^{\prime \prime}$ с векторами $q^{\prime \prime}=\left(q_{i}, q_{\alpha}^{\prime}\right)^{*}, \quad Q^{\prime \prime}=\left(Q_{i}{ }^{1}, Q_{\alpha}^{\prime}\right)^{*}, \quad i=\overline{1, n}, \quad$ и лагранжианом $L\left(t, q^{\prime \prime}, d q^{\prime \prime} / d t\right)$, образованную системами $S^{1}:\left\{q=\left(q_{i}\right)^{*}, Q^{1}=\left(Q_{i}{ }^{1}\right)^{*}\right\}$ и $S^{\prime}:\left\{q^{\prime}=\left(q_{\alpha}^{\prime}\right)^{*}, Q^{\prime}=\left(Q_{\alpha}^{\prime}\right)^{*}\right\}, \alpha=\overline{n+1, n+m}$. Пусть известно движение $q^{\prime}=f(t)$ массивной подсистемы $S^{\prime}$, независимое от движений $S^{1}$. Цилиндр $q^{\prime}=f(t), q^{\prime}=f^{*}(t)$ - инвариантное множество $C$ движений $S^{\prime \prime}$. Обозначим $Q=Q_{0}{ }^{1}=Q^{1}\left(t, q, f, q^{*}, f^{*}\right), L_{0}^{\prime \prime}=L^{\prime \prime}\left(t, q, f, q^{*}, f^{*}\right)$. При $L_{0}^{\prime \prime} \equiv L_{1}$ и совпадении на $q^{\prime}=f(t)$ множеств $\{d q\},\{\delta q\}$ для $S^{1}$ и $S$ множества движений $S$ и $S^{1}$ (на цилиндре $C$ ) совпадают. Следовательно, $S^{1} \in S^{+}$на $C$, а функция $G_{1}$ - частный инвариант движений $S^{\prime \prime}$ на $C$, если удовлетворяются условия $(1.2),(1.4), W(Q) \equiv 0$.

Ниже ограничимся потенциальной голономной системой $S^{+}$вида

$$
q_{i}=\partial H_{1} / \partial p_{i}, \quad p_{i}=-\partial H_{1} / \partial q_{i} \quad\left(v_{0} \equiv 1, Q_{i} \equiv 0, i=\overline{1, n)},\right.
$$

$H_{1}(t, q, p)=G_{1}-v_{i} p_{i}, \quad G_{1}(t, q, p)=G(x, p), \quad x=q+u, \quad u_{i}=\int_{0}^{t} v_{i}(\tau) d \tau$.

Согласно (1.4), система (1.5) имеет инвариант $G_{1}$ и, обратно, если $G_{1}$ - инвариант системы (1.5), то она является системой $S^{+}$. Система (1.5) эквивалентна автономной канонической системе с гамильтонианом $G(x, p)$. Действительно, преобразование $x=\partial F / \partial p^{\prime}, \quad p=\partial F / \partial q=p^{\prime}$, $F=p^{\prime} \cdot(q+u)$ переводит (1.5) в автономную каноническую систему с гамильтонианом - инвариантом вида

$$
x=\partial G / \partial p, \quad p^{\prime}=-\partial G / \partial x\left(G=G(x, p)=H^{\prime}=H_{1}+\partial F / \partial t\right) .
$$

Следовательно, система (1.5) с инвариантом $G_{1}(t, q, p)$ преобразуется в автономную систему (1.6), для которой имеем равенства

$$
\begin{gathered}
L_{1}\left(t, q, q^{*}\right)=L(x, x)=p \cdot x-G(x, p), \quad p=\partial L_{1} / \partial q=\partial L / \partial x, \quad \\
A^{-1}=B=B^{*} \quad\left(A=\left\|\partial^{2} L / \partial x_{i} \partial x_{k}\right\|, B=\left\|\partial^{2} G / \partial p_{i} \partial p_{k}\right\|, \quad i, k=\overline{1, n)} .\right.
\end{gathered}
$$

Система $S^{+}$вида (1.5) эквивалентна (1.6), допускающей понижение порядка на две единицы. Введем переменную $p_{0}=-G(x, p)$. При $x_{n}{ }^{\cdot}=\partial G / \partial p_{n} \neq 0$ обозначим решение уравнения $p_{0}=-G\left(x_{i}, p_{j}, p_{n}\right)$ $(j=\overline{1, n-1})$ через $P\left(x_{i}, p_{j}, p_{0}\right)=-p_{n}$. Тогда для определения траекторий имеем $2(n-1)$-мерную каноннческую систему и уравнение зависимости $x, p$ от $t$ вида

$x_{j}^{\prime}=\partial P / \partial p_{j}, p^{\prime}{ }_{j}=-\partial P / \partial x_{j}, t^{\prime}=\partial P / \partial p_{0}\left(p^{\prime}{ }_{0}=-\partial P / \partial t=0, f^{\prime}=d f / d x_{n}\right)$.

При $x_{i}{ }^{\alpha}=x_{i}\left(t_{\alpha}\right)=$ const и нефиксированных $t_{0}, t_{1}$ на траектории $y(\tau)$ системы

$$
d\left(\partial K / \partial x_{j}^{\prime}\right) / d \tau=\partial K / \partial x_{j}
$$

$$
t^{\prime}=-\partial K / \partial p_{0} \quad\left(y=\left(x_{j}\right)^{*}, \tau=x_{n}, p_{0}=\text { const, } \alpha=0,1\right) \text {, }
$$




$$
\begin{aligned}
& K\left(x, y^{\prime}, p_{0}\right) \equiv x_{j}^{\prime} P_{j}\left(x, y^{\prime}, p_{0}\right)-P\left(x, P^{1}, p_{0}\right) \\
& \left(x=\left(x_{j}, \tau\right)^{*}, \quad P^{1}=\left(P_{j}\right)^{*}, \quad j=\overline{1, n-1)},\right.
\end{aligned}
$$

полученной из (1.8) решением уравнений $x_{j}^{\prime}=\partial P / \partial p_{j}$ относительно $p_{j}$ в пересечении областей определения $P$ и $\left|\partial^{2} P / \partial p_{j} \partial p_{k}\right| \neq 0$, стационарно действие типа Якоби

$$
W=\int_{\tau_{0}}^{\tau_{1}} K\left(y, \tau, y^{\prime}, p_{0}\right) d \tau \quad\left(\tau_{\alpha}=x_{n}\left(t_{\alpha}\right), y(\tau) \in C_{2}\right) .
$$

В случае $B=\left\|\partial^{2} G / \partial p_{s} \partial p_{\sigma}\right\|>0$ на $R_{0}$ для получения $K$ исключим $t^{\prime}$ из (1.9) с помощью $G$

$$
K=t^{\prime} x_{i} p_{i}=t^{\prime}(L+G)=t^{\prime}\left(L-p_{0}\right)=t^{\prime}\left[L^{1}\left(x, y^{\prime}, t^{\prime}\right)-p_{0}\right]\left(x_{i}=x_{i}^{\prime} / t^{\prime}\right),
$$

$L^{1}\left(x, y^{\prime}, t^{\prime}\right) \equiv L\left(x, y^{\prime} / t^{\prime}, 1 / t^{\prime}\right), \quad p_{n}=-P, \quad R_{0}=\left\{\partial G / \partial p_{n} \neq 0, \quad G=-p_{0}\right\}$

$$
(i, s, \sigma=\overline{1, n}) \text {, }
$$

$$
\begin{gathered}
-G \equiv f\left(x, y^{\prime}, t^{\prime}\right)=L^{1}\left(x, y^{\prime}, t^{\prime}\right)+t^{\prime} \partial L^{1} / \partial t^{\prime}=L-x_{i} \partial L / \partial x_{i}=p_{0}, \\
b_{s \sigma}=\partial^{2} G / \partial p_{s} \partial p_{\sigma}=\partial^{2} H / \partial p_{s} \partial p_{\sigma} .
\end{gathered}
$$

Из $(1.7),(1.8),(1.10)$ в области $R_{0}$ находим неравенства

$1 / t^{\prime}=\partial G / \partial p_{n} \neq 0, \quad A=B^{-1}>0, \quad t^{\prime} \partial f / \partial t^{\prime}=\partial G / \partial p \cdot A \partial G / \partial p>0, \quad \partial f / \partial t^{\prime} \neq 0$,

при которых уравнение $f=p_{0}$ имеет в некоторой окрестности точки $\left(x_{0}, y_{0}^{\prime}, p_{0}\right) *$ единственное решение $t^{\prime}=T\left(x, y^{\prime}, p_{0}\right) \in C_{1}$. Подстановкой $t^{\prime}=T$ в (1.10) находим $K\left(x, y^{\prime}, p_{0}\right)$, которая является функцией Рауса для лагранжиана $L_{1}^{1}\left(x, y^{\prime}, t^{\prime}\right) \equiv t^{\prime} L^{1}\left(x, y^{\prime}, t^{\prime}\right)$. Действительно, из (1.10), (1.11) имеем

$$
R\left(x, y^{\prime}, p_{0}\right)=L_{1}^{1}-t^{\prime} \partial L_{1}^{1} / \partial t^{\prime}=L_{1}^{1}-p_{0} t^{\prime}=K\left(x, y^{\prime}, p_{0}\right) .
$$

Из инвариантности экстремалей при замене переменных найдем для (1.5) две формы модификации принципа наименьшего действия. На движении $\left(t\left(x_{n}\right), q_{j}\left(x_{n}\right)\right)^{*}$ интеграл

$$
\begin{gathered}
W_{1}=\int_{\tau_{0}}^{\tau_{1}} K^{1}\left(t, \xi, \tau, t^{\prime}, \xi^{\prime}, p_{0}\right) d \tau\left(\xi=\left(q_{j}\right)^{*}, \xi^{\prime}=d \xi / d \tau, d t / d \tau=t^{\prime}\right), \\
K^{1}=K\left(\xi+w, \tau, \xi^{\prime}+t^{\prime} w^{*}, p_{0}\right)\left(\xi, t \in C_{2}(\tau), w=\right. \\
=\left(u_{j}\right)^{*}, \tau=x_{n}=q_{n}+u_{n}(t), j=\overline{1, n-1)}
\end{gathered}
$$

имеет стационарное значение для всех кривых, удовлетворяющих условиям

$$
\begin{gathered}
q_{j}\left(\tau_{\alpha}\right)+u_{j}\left[t\left(\tau_{\alpha}\right)\right]=c_{j \alpha}^{1}=\text { const } \\
\left(\tau_{\alpha}=\text { fixconst, } c_{\alpha}^{1}=y\left(\tau_{\alpha}\right), q_{j}^{\prime}=d q_{j} / d \tau, \alpha=0,1\right), \\
L^{0}+t^{\prime}\left(\partial L^{0} / \partial t^{\prime}-u_{j} \partial L^{0} / \partial q_{j}^{\prime}\right)= \\
=p_{0}\left(L^{0}\left(t, \xi, \tau, t^{\prime}, \xi^{\prime}\right)=L_{1}\left(t, q_{j}, \tau-u_{n}, q_{j}^{\prime} / t^{\prime}, 1 / t^{\prime}-u_{n}\right) .\right.
\end{gathered}
$$

Обозначим $G^{1}\left(x, x^{*}\right)=G\left(x, \partial L / \partial x^{*}\right)$. Из (1.10), используя $\Delta$-вариа- 
цию $(\Delta F=\delta F+\Delta t d F / d t)$, находим стационарность действия типа Лагранжа

$$
W_{2}=\int_{t_{0}}^{t_{t}} x_{i} \partial L / \partial x_{i} d t \quad\left(F=F(t, x, x), \partial L / \partial x_{i}=p_{i}, i=\overline{1, n}\right)
$$

при $\quad G^{1}[x(t), x(t)] \equiv x_{i} \partial L / \partial x_{i}-L(x, x)=-p_{0}, x_{i}\left(t_{\alpha}\right)=x_{i \alpha}=$ const на движении $x(t)$ системы (1.6). Действительно, из (1.7), (1.12) получаем равенства

$$
\begin{gathered}
\Delta G^{1}=0,\left.\quad(p \cdot \Delta x)\right|_{0} ^{1} \equiv p\left(t_{1}\right) \cdot \Delta x\left(t_{1}\right)-p\left(t_{0}\right) \cdot \Delta x\left(t_{0}\right)=0, \\
\Delta W_{2}=\Delta \int_{t_{0}}^{t_{1}}(L+G) d t=\Delta \int_{t_{0}}^{t_{1}} L d t-p_{0} \Delta\left(t_{1}-t_{0}\right)=\left.(p \cdot \Delta x)\right|_{0} ^{1}=0,
\end{gathered}
$$

которые доказывают утверждение. В переменных $t, q$ из (1.12), (1.13) имеем $\Delta W_{3}=0, \quad W_{3}=\int_{t_{0}}^{t_{s}}\left(q_{i}+u_{i}\right) \partial L_{1} / \partial q_{i} d t\left(q_{i}=d q_{i} / d t, \quad i=\overline{1, n}, \quad \alpha=0,1\right)$ при условиях $\left(q^{*}+u\right) \cdot \partial L_{1} / \partial q-L_{1}=-p_{0}, \quad q\left(t_{\alpha}\right)+u\left(t_{\alpha}\right)=$ $=$ const $\left(u \cdot\left(t_{\alpha}\right) \Delta t_{\alpha}=-\Delta q\left(t_{\alpha}\right)\right)$.

2. Обозначим невозмущенное и возмущенное движения системы (1.6) через $x^{1}(t), p^{1}(t), \xi=x-x^{1}, \eta=p-p^{1}$ соответственно. Из равенств $q^{1}=x^{1}-u, \xi=q-q^{1}$ заключаем, что исследование устойчивости решения (1.5) по всем (некоторым) переменным сводится к аналогичной задаче для движения $x^{1}, p^{1}$-автономной системы (1.6). При этом точкам покоя (1.6) отвечают нестационарные движения (1.5), если $u \neq \equiv$. Қанонические уравнения возмущений (1.5)

$$
\begin{gathered}
\xi=\partial G^{1} / \partial \eta, \quad \eta=-\partial G^{1} / \partial \xi, \\
G^{1}=G\left(x^{1}+\xi, p^{1}+\eta\right)-\xi \cdot \partial G / \partial x^{1}-\eta \cdot \partial G / \partial p^{1}-G\left(x^{1}, p^{1}\right)
\end{gathered}
$$

имеют инвариант $V^{1}(t, \xi, \eta)=G\left(x^{1}+\xi, p^{1}+\eta\right)-G\left(x^{1}, p^{1}\right)$. Приведем достаточные условия устойчивости стационарных движений $x^{1}=$ $=x^{0}=$ const, $p^{1}=p^{0}=$ const, заданных равенствами

$$
\partial G / \partial x^{0}=\partial G / \partial p^{0}=0 \quad\left(x^{0}=\left(x_{i}^{0}\right)^{*}, p^{0}=\left(p_{j}^{0}\right)^{*}, i, j=\overline{1, n)} .\right.
$$

В новых обозначениях инвариант $V^{1}$ системы (2.1) имеет вид

$$
V^{1}=V(z)=G^{1}(z)=G\left(z^{0}+z\right)-G\left(z^{0}\right), \quad V(0)=0,
$$

$z=\left(\xi_{\alpha}, \xi_{\beta}, \eta_{j}\right)^{*}, \quad z^{0}=\left(x_{i}^{0}, p_{j}^{0}\right)^{*}, \quad y=\left(\xi_{\beta}, \eta_{j}\right)^{*}, \quad \xi^{\prime}=\left(\xi_{\alpha}\right)^{*}, \quad \xi^{\prime \prime}=\left(\xi_{\beta}\right)^{*}$,

$$
\alpha=\overline{1, k} \leqslant n, \quad \beta=\overline{k+1, n}, \quad k \geqslant 0, \quad i, j=\overline{1, n} .
$$

Пусть $V(z)$ - $y$-определенно положительная функция. Тогда $\exists W \in C(y), W(0):=0, W>0, y \neq 0, V \geqslant W$ на $|y| \leqslant \delta_{0}=$ const и стационарное движение $x^{0}, p^{0}$ устойчиво [ $\left.{ }^{1}\right]$ по переменным $x_{\beta}, p_{i}$. Если $S^{+}$- натуральная система, то инвариант (2.2) системы (2.1) будет определенно положительным по $\xi_{\beta}, \eta_{i}{ }^{1}$

$V(z)=1 / 2 \eta^{1} B \eta^{1}+P(x)-P\left(x^{0}\right), \eta^{1}=\eta+g(\xi), g=A(x) b(x)-A\left(x^{0}\right) b\left(x^{0}\right)$,

$$
\begin{array}{cc}
P(x)=b_{0}(x)-1 / 2 b(x) \cdot A(x) b(x), & g(0)=0, p^{0}=-A\left(x^{0}\right) b\left(x^{0}\right), \quad x=x^{0}+\xi, \\
B=A^{-1}=\left\|b_{i j}\left(x^{0}+\xi\right)\right\|, & G(x, p)=1 / 2 b_{i j} p_{i} p_{j}+b_{i} p_{i}+b_{0}
\end{array}
$$


при $\quad \eta^{1} \cdot B \eta^{1} \geqslant b^{0}\left|\eta^{1}\right|^{2} \equiv W_{1}\left(\eta^{1}\right), \quad P(x)-P\left(x^{0}\right) \geqslant W_{0}\left(\xi^{\prime \prime}\right) \quad\left(0<b^{0}=\right.$ const $)$, $W_{0}>0, \quad \xi^{\prime \prime} \neq 0, \quad W_{0}(0)=0, \quad W_{0}\left(\xi^{\prime \prime}\right) \in C, \quad 0 \leqslant\left|\xi^{\prime \prime}\right| \leqslant \delta_{0}=$ const. $\quad$ Здесь $V \geqslant W \equiv W_{0}\left(\xi^{\prime \prime}\right)+W_{1}\left(\eta^{1}\right)$. Пусть $g_{i}(\xi) \rightarrow 0$ при $\left|\xi^{\prime \prime}\right| \rightarrow 0$ равномерно по $\xi^{\prime}$, а $g_{i}\left(\xi^{\prime}, 0\right) \equiv 0$. Тогда из неравенств $\left|\eta_{i}\right| \leqslant\left|\eta_{i}{ }^{1}\right|+\left|g_{i}\right|$ и непрерывности $g_{i}(\xi)$ следует устойчивость $x^{0}, p^{0}$ по переменным $x_{\beta}, p_{i i}$. Если $z^{0}$ - изолированный минимум $G$, то $x^{0}, p^{0}$ устойчиво по всем переменным. Этот случай имеем при $\left\|\partial^{2} G / \partial z_{s}{ }^{0} \partial z_{\sigma}{ }^{0}\right\|>0$, где $n$ неравенств выполняются в силу $B>0, z^{0}=\left(x_{i}{ }^{0}, p_{j}{ }^{0}\right) *, s, \sigma=\overline{1,2 n}$. Рассмотрим $x^{0}, p^{0}$-устойчивость Лагранжа по переменным $x_{\beta}, p_{i}$. Пусть при равномерности по $\xi^{\prime} \in E^{k}$ имеем

$$
G^{1}(z) \geqslant W^{1}(y), W^{1} \rightarrow+\infty \text { при }|y| \rightarrow \infty \quad\left(W^{1}(y) \in C\left(E^{2 n-k}\right)\right) .
$$

Тогда для любого решения $(2.1)$ с началом в $\left|y\left(t_{0}\right)\right| \leqslant$ const предположение $\lim |y(t)|=\infty$ противоречит условиям (2.4) и $G^{\mathrm{L}}\left[\xi^{\prime}(t), y(t)\right]=$ $=$ const. Следовательно, $x^{0}, p^{0}$ относительно $x_{\beta}, p_{i}$ устойчиво по Лагранжу. С учетом (2.3) условие (2.4) выполняется для натуральной (1.5), если

$\min (e \cdot B e) \geqslant \beta_{0}=$ const, $\quad|e|=1 ; \quad|B g| \leqslant \gamma_{0}=$ const $\quad(g=g(\xi))$,

$$
P\left(x^{0}+\xi\right) \geqslant P_{1}\left(\xi^{\prime \prime}\right) \in C ; \quad P_{1}\left(\xi^{\prime \prime}\right) \rightarrow+\infty,\left|\xi^{\prime \prime}\right| \rightarrow \infty \quad\left(\xi \in E^{n}\right) .
$$

$y$-компонента любого решения (2.1) ограничена, так как в случае (2.5) функция $W_{0}^{1}(y)=1 / 2 \beta_{0}|\eta|^{2}-\gamma_{0}|\eta|+P_{1}\left(\xi^{\prime \prime}\right)-P\left(x^{0}\right)$ удовлетворяет (2.4).

3 а м е ан ие 2. Все ранее сказанное в пп. 1 и 2 справедливо и для случая неавтономной системы Гамильтона $S_{+}$с инвариантом $G_{+}=$ $=H(t, q, p)+h \cdot p$, где $h=\left(h_{i}\right)^{*}=h\left(q_{\alpha}\right), \quad h_{\alpha} \neq 0, \quad \partial h / \partial q_{\beta} \equiv 0, \quad \beta \neq$ $\neq \alpha \in 1, n$. Действительно, вводя переменные $x, p^{\prime}$ равенствами

$$
\begin{gathered}
x=\partial f / \partial p^{\prime}, p=\partial f / \partial q, f \equiv g \cdot p=\sum_{s=1}^{n} g_{s} p_{s}, \\
g_{\alpha}=t+\int_{q_{\alpha}^{0}}^{q_{\alpha}} h_{\alpha}^{-1}(\xi) d \xi, \quad g_{\beta}=q_{\beta}-\int_{q_{\alpha}^{0}}^{q_{\alpha}} h_{\alpha}^{-1}(\xi) h_{\beta}(\xi) d \xi \quad(\alpha \neq \beta=\overline{1, n)},
\end{gathered}
$$

получим аналогичную (1.6) автономную систему

$$
d x / d t=\partial G / \partial p^{\prime}, \quad d p^{\prime} / d t=-\partial G / \partial x \quad\left(G=G\left(x, p^{\prime}\right)=G_{+}(t, q, p)\right) .
$$

3. Примеры. С учетом замечания 1 рассмотрим аналогичную $\left[{ }^{2}\right]$ систему $S^{1}$, состоящую из воздействующей $S^{\prime}$-системы $M$ центров, вращающихся вокруг о $\beta$ с $\Omega_{0}=$ const $\geqslant 0, \quad r_{v}=\Omega_{0} \beta \times r_{v} \quad(v=\overline{1, M})$, и свободной системы из $N$ точек $S^{+}$с функцией внутренних и внешннх сил - потенциалом позиционных и электромагнитных взаимодействий вида

$$
\begin{gathered}
L_{0}\left(r^{\prime}\right)+\sum_{s=1}^{N} a_{s}\left(r^{\prime}\right) \cdot r_{s}^{\prime}, \\
L_{0}=\sum_{v, s}^{M, N} f_{v s}(\varrho v \alpha, \varrho s \alpha, \varrho v s)+\sum_{s \neq \sigma}^{N} f_{s \sigma}(\varrho v \alpha, \varrho \sigma \alpha, \varrho s \sigma)(\alpha=1,2), \\
\varrho_{i 1}=\left|r_{i}\right|, \quad \varrho_{i 2}=r_{i} \cdot \beta, \quad \varrho_{i j}=\left|r_{i}-r_{j}\right|, \\
r^{\prime}=\left(r_{s}^{\prime}\right)^{*}, \quad r_{s}^{\prime *}=r_{s}-\Omega_{0} \beta \times r_{s}, \quad \beta=\text { const, } \quad|\beta|=1 ; \quad s, \sigma=\overline{1, N} .
\end{gathered}
$$


В силу результатов пп. 1 и 2 , система $S^{+}$имеет инвариант движения

$$
\begin{gathered}
G\left(r^{\prime}, p^{\prime}\right)=1 / 2 m_{s} r_{s}^{2}-L_{0}-\Omega_{0} \beta \cdot\left(M++m^{+}\right), \\
M^{+}=r_{s} \times m_{s} r_{s}, \quad m^{+}=r_{s}^{\prime} \times a_{s}^{\prime} .
\end{gathered}
$$

Подслучаем этого случая будет гиростат $S$ Жуковского, если $S^{\prime}$ массивный гиростат $S_{0}\left(M_{1} / M_{0} \ll 1\right)$, вращающийся вокруг $\beta$ с $\Omega_{0}=$ $=$ const $\geqslant 0$. Ниже для простоты примем $L_{1}=a^{\prime}\left(r^{\prime}\right) \cdot r^{\prime \cdot}+a^{\prime \prime}\left(\lambda^{\prime}\right) \omega$ $\left(\omega^{\prime}=\omega-\Omega_{0} \beta\right)$, где $r^{\prime}, \lambda^{\prime}-$ координаты центра масс и ориентации $S_{1}$ относительно неподвижного в $S_{0}$ триэдра $о e_{1} e_{2} \beta$. Из (3.1) с учетом обозначений $\left[{ }^{2}\right]$ находим инвариант поступательно-вращательного движения $S_{1}$ относительно $S_{0}$ в виде его гамильтониана

$$
\begin{gathered}
H=G\left(r^{\prime}, \lambda^{\prime}, p\right)=H^{\prime}\left(r^{\prime}, p^{\prime}, \lambda^{\prime}\right)+H^{\prime \prime}\left(\lambda^{\prime}, p^{\prime \prime}, r^{\prime}\right)-V\left(r^{\prime}, \lambda^{\prime}\right) \\
\left(p=\left(p_{i}^{\prime}, p_{j}^{\prime \prime}\right)^{*}, \quad i, j=\overline{1,3}, \quad Q_{k} \equiv 0\right),
\end{gathered}
$$

$$
\begin{gathered}
H^{\prime}=1 / 2 M_{1}\left[r^{\prime \cdot 2}-\Omega_{0}^{2}\left(\beta \times r^{\prime}\right)^{2}\right]+V \quad\left(\omega=\omega^{\prime}+\Omega_{0} \beta, r=r^{\prime}+\Omega_{0} \beta \times r\right), \\
H_{(\beta)}^{\prime \prime}=T_{2}+V-\Omega_{0} \beta \cdot K, \quad 2 T_{2}=\omega \cdot C \omega+I_{h}^{-1} r^{2}{ }_{h}, \\
\left.K=G \omega+k+a^{\prime \prime}, \quad C=G-g \overline{\left(00_{1}\right.}=r\right), \\
r_{k}=I_{k}\left[\left(\omega \cdot i_{k}\right)+a_{k}\right]=\mathrm{const}, \\
g=\left\|g_{\alpha \beta}\right\|, \quad g_{\alpha \beta}=\sum_{k=1}^{\rho} I_{k}\left(i_{k \alpha} \cdot i_{k \beta}\right)(\alpha, \beta=\overline{1,3}, k=1, \varrho), \\
k=\sum_{k=1}^{\rho} I_{k} \dot{\alpha}_{k} i_{k}, \quad l=g \omega+k=\sum_{k=1}^{\rho} r_{k} i_{k}, \quad L=1 / 2 M_{1} r^{2}+T_{2}+L_{1}-V, \\
p^{\prime}=\partial L / \partial r^{\prime}, \quad p^{\prime \prime}=\partial L / \partial \lambda^{\prime} ;
\end{gathered}
$$

где $i_{k}$ - орты твердых и жидких роторов, фиксированных в $o_{1} e_{1}^{\prime} e_{2}{ }_{2} e_{3}-$ главном триэдре $S_{1}$. Из допущения у $S_{1}$ инвариантных множеств $r^{\prime}=r^{\prime} \cdot[t], r^{\prime \cdot}=r_{*}^{\prime}[t]$ в ограниченной задаче при $V_{*} \neq V_{0}{ }^{*}[t]+V_{1}{ }^{*}\left(\lambda^{\prime}\right)$ следует ошибочный вывод о существовании на $r^{\prime}=r^{\prime}$. инварианта вращения $S_{1}$ в виде $H_{*}^{\prime \prime}+T^{\prime} *[t] \quad\left(H_{*}^{\prime \prime} \equiv H^{\prime \prime}\left|r^{\prime}=r_{*}^{\prime}, \quad T_{*}^{\prime}=T^{\prime}\right| r^{\prime}=r_{*}^{\prime}\right)$ Аналогичные пп. 1,2 и замечанию 2 результаты $\left[{ }^{3}\right]$ получим для подсистемы вращения $S_{1}{ }^{*}$ с инвариантом $G=g_{0} H_{*}{ }^{\prime \prime}+g_{i} p_{i *}{ }^{\prime \prime}$ вращения $S_{1}$ при $r^{\prime}=r_{*}^{\prime}[t] \quad\left(g_{j}=g_{j}\left(t, \lambda^{\prime}\right), j=\overline{0,3}\right)$. В частности, рассмотрим круговое движение центра масс $S_{1} \quad\left(\left|r_{*}\right|=r_{0}=\right.$ const, $\omega_{r}=\omega_{0} n=$ const, $\omega_{0}=$ const, $\left.M_{1} / M_{0} \ll 1\right)$, когда оси симметрин o $\beta$ и вращения с $\Omega_{0}(t) \sim 0$ гравитирующего сфероида $S_{0}$ совпадают и выполняются условия

$$
\varepsilon_{1}=R_{1} r_{0}^{-1} \ll 1, \quad\left|\varepsilon_{2}\right| \equiv\left|C_{0}-A_{0}\right| M_{0}^{-1} R_{0}^{-2} \ll 1, \quad a^{\prime \prime}=a^{\prime \prime}(n, \gamma) .
$$

При $\widetilde{\nabla}$-асимптотике $V$ * с $\varepsilon_{1}{ }^{2}, \varepsilon_{2}$-членами $\left[{ }^{2}\right]$ из $(3.2)$ находим $G$-инвариант

$$
\begin{aligned}
& H_{n^{*}}^{\prime \prime} \simeq G=T_{2}+\widetilde{V}^{1}-\omega_{0} n \cdot K, \quad L^{\prime \prime}=T_{2}+a^{\prime \prime} \cdot \omega-V_{*}\left(K=G_{\omega}+k+a^{\prime \prime}\right), \\
& -\widetilde{\nabla}=\mu r_{0}^{-1}\left\{M_{1}\left[1-\frac{\varepsilon_{2} R_{0}^{2}\left(1-3 s^{2}\right)}{2 r_{0}^{2}}\right]-\frac{3 \varepsilon_{1}^{2}}{2 R_{1}^{2}}\left[\gamma \cdot G \gamma-1 / 3 \delta_{i j} G_{j}\right]\right\}(i, j=\overline{1,3}) .
\end{aligned}
$$


$s^{2}(t)=\sin ^{2} \alpha_{0} \cos ^{2}\left[\omega_{0}\left(t-t_{0}\right)+c_{0}\right], \cos \alpha_{0} \equiv n \cdot \beta, \quad \gamma=\gamma_{i} e_{i}^{\prime}=r / r_{0},\left.\widetilde{\nabla}_{*}^{1} \equiv \tilde{V}_{*}\right|_{s=0}$.

3 а м еч ан и е 3 . При круговом движении $r *[t]$ и произвольной функции $V\left(t, r^{\prime}, \lambda^{\prime}\right)$ для инвариантности $H_{n *^{\prime \prime}}$ необходимо и достаточно, чтобы $V_{*}=V_{0}^{*}[t]+V_{1}{ }^{*}(\gamma, n)$. Отсюда в случае трехосного $S_{0}$ при главном резонансе $\beta=n, \Omega_{0}=\omega_{0}$ сохраняется гамильтониан вращения $S_{1}$ относительно орбитной системы координат $o_{1} \gamma \tau n$.

$$
H_{*}^{\prime \prime}=G=T_{2}-\omega_{0} \beta \cdot K+V *\left(V *=\left.V_{1}^{*}(\gamma, \beta) \equiv V\left(r^{\prime}, \lambda^{\prime}\right)\right|_{r=r \cdot}, V_{0}^{*} \equiv 0\right)
$$

В связи с (3.2), (3.3) рассмотрим систему, для которой $H=H(\zeta, \varepsilon)-$ голоморфная по $\varepsilon$ функция; решения и инвариант $H-\varepsilon$-аналитичны и представимы в $t \geqslant t_{0},|\zeta|<\infty$ равномерно сходящимися рядами

$$
\begin{gathered}
\zeta=\sum_{k=0}^{\infty} \varepsilon^{k} \zeta_{k}(t), \quad H(\xi, \varepsilon)=\sum_{k=0}^{\infty} \varepsilon^{k} H_{k}\left(t, \zeta_{k}\right), \\
H=H_{0}^{\prime}(x, p)+H_{0}^{\prime \prime}(\xi, \psi)+\varepsilon^{2} V(x, \xi, \varepsilon), \\
H_{0}=H_{0}^{\prime}+H_{0}^{\prime \prime}, \quad V=\sum_{k=0}^{\infty} \varepsilon^{k} V_{k}(x, \xi), \quad \xi=\left(y_{s}, z_{\sigma}\right)^{*}, \\
y=\left(x_{i}, p_{j}\right)^{*}, \quad z=\left(\xi_{i}, \psi_{j}\right)^{*} \quad(s, \sigma=\overline{1,2 n} ; i, j=\overline{1, n}) .
\end{gathered}
$$

Так как при $k \geqslant 1$ в (3.2), (3.3) $V_{k}(x, \xi)$ плохо известны, то имеет смысл ограничиться $\varepsilon^{2}$-приближениями $\quad \tilde{\zeta}_{2} \equiv \zeta_{0}+\varepsilon^{2} \xi_{2} \simeq \zeta, \quad \tilde{H}_{2} \equiv$ $\equiv H_{0}+\varepsilon^{2} H_{2} \simeq H$, где

$x_{0}=\partial H^{\prime}{ }_{0} / \partial p_{0}, \quad p_{0}=-\partial H_{0}^{\prime} / \partial x_{0}, \quad \xi_{0}=\partial H_{0}^{\prime \prime} / \partial \psi_{0}, \quad \psi_{0}^{*}=-\partial H_{0}^{\prime \prime} / \partial \xi_{0}$,

$$
x_{2}=\partial l^{\prime}{ }_{0} / \partial p_{0},
$$

$$
\begin{gathered}
p_{2}=-\partial l_{0}^{\prime} / \partial x_{0}-k \sim y_{2}=A y_{2}+f\left(l_{0}^{\prime} \equiv y_{2} \cdot \nabla H_{0}^{\prime}\left(y_{0}\right), k \equiv \partial V_{0} / \partial x_{0}\right), \\
\xi_{2}=\partial l_{0}^{\prime \prime} / \partial \psi_{0},
\end{gathered}
$$

$$
\psi_{2}=-\partial l_{0}^{\prime \prime \prime} / \partial \xi_{0}-h \sim z_{2}=B z_{2}+g \quad\left(l_{0}^{\prime \prime} \equiv z_{2} \cdot \nabla H_{0}^{\prime \prime}\left(z_{0}\right), h \equiv \partial V_{0} / \partial \xi_{0}\right) .
$$

Пусть (как в случае (3.3), $a^{\prime \prime}=$ const) для (3.5) известно решение $\zeta_{0}\left[t, \zeta_{0}\left(t_{0}\right)\right]$. Тогда, согласно теореме Пуанкаре, известны матрицанты, $\xi_{2}$-решение и реальный $\varepsilon^{2}$-асимптотический инвариант достоверной части системы (3.4)

$$
\begin{gathered}
y_{2}=Y y_{20}-\int_{t_{0}}^{t} Y_{2}^{-} k d \tau, \quad Y\left(t_{0}, t_{0}\right)=I, \quad Y-1=\left\|Y_{1}^{-} Y_{2}^{-}\right\|, \quad \xi_{2}\left(t_{0}\right)=\xi_{20}, \quad X X-1=I, \\
z_{2}=Z z_{20}-\int_{t_{0}}^{t} Z_{2}^{-} h d \tau, \quad Z\left(t_{0}, t_{0}\right)=I, \quad Z^{-1}=\left\|Z_{1}^{-}, Z_{2}^{-}\right\|, \\
\zeta\left(t_{0}\right)=\xi_{0}\left(t_{0}\right), \quad \zeta_{2}^{0}=\left.\xi_{2}\right|_{h=0} ^{k=0}, \\
H=H_{0}\left(\zeta_{0}\right)+\varepsilon^{2}\left[\xi_{2} \cdot \partial H_{0} / \partial \xi_{0}+V_{0}\left(x_{0}, \xi_{0}\right)\right]+o\left(\varepsilon^{2}\right)= \\
=H_{0}\left(\tilde{\xi}_{2}\right)+\varepsilon^{2} V_{0}\left(x_{0}, \xi_{0}\right)+o\left(\varepsilon^{2}\right),
\end{gathered}
$$

$H_{0}\left[\zeta_{0}(t)\right]=$ const; $\quad \xi_{2}^{0} \cdot \partial H_{0} / \partial \xi_{0}=$ const,$\quad H \simeq \tilde{H}_{2} \equiv H_{0}\left(\tilde{\zeta}_{2}\right)+\varepsilon^{2} V_{0}\left(\tilde{x}_{2}, \tilde{\xi}_{2}\right)$, 


$$
P(t)-P\left(t_{0}\right)=\left.\left(x_{0} \cdot \partial V_{0} / \partial x_{0}\right)\right|_{t} ^{t_{0}}+\int_{t_{0}}^{t} x_{0} \cdot\left(\partial V_{0} / \partial x_{0}\right) \cdot d \tau, \quad P \equiv y_{2} \cdot \partial H^{\prime}{ }_{0} / \partial y_{0}
$$

Из (3.5), (3.6) выводим необходимое и достаточное условие независимости $\widetilde{y}_{2}$ от $\tilde{z}_{2}$ (или $\tilde{z}_{2}$ от $\widetilde{y}_{2}$ ), эквивалентное $\varepsilon^{2}$-инвариантности $y$ (или $z$ ) вида

$$
\partial^{2} V_{0} / \partial \xi \partial x_{0}=\partial\left[\partial V_{0}\left(\xi, x_{0}\right) / \partial x_{0}\right] / \partial \xi \equiv 0\left(\partial^{2} V_{0} / \partial x \partial \xi_{0} \equiv 0\right) .
$$

Вдоль $x_{0}(t)$, удовлетворяющего (3.7), согласно (3.6) $z$-подсистема (3.4) имеет $\varepsilon^{2}$-асимптотический инвариант $H_{0}^{\prime \prime}(\xi, \psi)+\varepsilon^{2} V_{0}\left[x_{0}(t), \xi\right]+H_{0}^{\prime}\left(\tilde{y}_{2}\right)$.

Рассмотрим устойчивость $v$-стационарного вращения $o_{1} e^{\prime}{ }_{i}$ вокруг $o_{1} \gamma \tau n$ в случае (3.3), где $V=V_{0}{ }^{*}[t]+V_{1}^{*}(\gamma, n), a^{\prime \prime}=$ const. Ориентацию $o_{1} e^{\prime}{ }_{i}$ к $o_{1} \gamma \tau n$ зададим матрицей $\left\|\alpha_{i j}[\lambda(v)]\right\|$, где $v_{i}$ - новые переменные без особенности в $\theta=2 \pi N$, для которых $V_{1}{ }^{*} \equiv P\left(\alpha_{i j}\right) / P_{0}\left(\alpha_{i j}\right)$ переходит в дробно-рациональную функцию от $v=\left(v_{i}\right)^{*}$ :

\begin{tabular}{c|cccc} 
& $\gamma$ & $\tau$ & $n$ & \\
$e_{1}^{\prime}{ }_{1}$ & $\lambda_{0}^{2}+\lambda_{1}^{2}-\lambda_{2}^{2}-\lambda_{3}^{2}$ & $2\left(\lambda_{0} \lambda_{3}+\lambda_{1} \lambda_{2}\right)$ & $2\left(\lambda_{1} \lambda_{3}-\lambda_{\theta} \lambda_{2}\right)$ & $\lambda_{0}=\left(|v|^{2}-1\right)\left(1+|v|^{2}\right)^{-1}$ \\
$e_{2}^{\prime}$ & $2\left(\lambda_{1} \lambda_{2}-\lambda_{0} \lambda_{3}\right)$ & $\lambda_{0}^{2}+\lambda_{2}^{2}-\lambda_{1}^{2}-\lambda_{3}^{2}$ & $2\left(\lambda_{0} \lambda_{1}+\lambda_{2} \lambda_{3}\right)$ & $\lambda_{j}=2 v_{j}\left(1+|v|^{2}\right)^{-1}$ \\
$e^{\prime}{ }_{3}$ & $2\left(\lambda_{0} \lambda_{2}+\lambda_{1} \lambda_{3}\right)$ & $2\left(\lambda_{2} \lambda_{3}-\lambda_{0} \lambda_{1}\right)$ & $\lambda_{0}^{2}+\lambda_{3}^{2}-\lambda_{1}^{2}-\lambda_{2}^{2}$ & $i, j=1,3$
\end{tabular}

$v_{1}=v_{0} \sin \varphi_{1} \cos \varphi_{2}, \quad v_{2}=v_{0} \sin \varphi_{1} \sin \varphi_{2}, \quad v_{3}=v_{0} \cos \varphi_{1} \sin \varphi_{3}$,

$$
v_{0}=\left(1-\cos \varphi_{1} \cos \varphi_{3}\right)^{-1} \text {, }
$$

$$
2 \varphi_{1}=\theta, \quad 2 \varphi_{2}=\psi-\varphi, \quad 2 \varphi_{3}=\psi+\varphi .
$$

В переменных $\omega, v$ уравнения вращения $S_{1}$ и инвариант (3.3) имеют вид

$K^{*}+\omega \times K+B^{*} \nabla_{v} V=0, v=B \omega^{\prime}\left(K=\partial T / \partial \omega=C \omega+b, T \equiv T_{2}+a^{\prime \prime} \cdot \omega\right)$, (3.9) $4 B=\left\|\begin{array}{cc}v_{2}^{2}+v_{3}^{2}-v_{1}^{2}-1, & -2\left(v_{1} v_{2}+v_{3}\right),-2\left(v_{1} v_{3}-v_{2}\right) \\ 2\left(v_{3}-v_{1} v_{2}\right), & v_{1}^{2}+v_{3}^{2}-v_{2}^{2}-1,-2\left(v_{1}+v_{2} v_{3}\right) \\ -2\left(v_{2}+v_{1} v_{3}\right), & 2\left(v_{1}-v_{2} v_{3}\right),\left(v_{1}^{2}+v_{2}^{2}-v_{3}^{2}-1\right)\end{array}\right\| \begin{aligned} & \operatorname{det} B \neq 0, b=l+a^{\prime \prime}=c_{0}, \\ & \omega=\omega^{\prime}+\omega_{0} n, \\ & \left(\partial T / \partial \alpha_{k}\right)^{\cdot}=Q_{k} \equiv 0,\end{aligned}$

$$
\begin{gathered}
G=R_{2}+W=1 / 2\left[\omega^{\prime} \cdot C \omega^{\prime}+\sum_{k=1}^{\rho} r_{k}^{2} I_{k}^{-1}+\omega_{0}^{2} W_{0}(v, c)\right], \\
W_{0}=\omega_{0}^{-2} P-n \cdot C n-2(c \cdot n), \\
P=2 V_{1}^{*}(v), 2 \nabla_{*}^{1}=3 \omega_{0}^{2}(\gamma \cdot G \gamma),
\end{gathered}
$$

$c \equiv b / \omega_{0}, \quad \omega_{0}=\omega_{0}\left(r_{0}, \varepsilon_{2}, \alpha_{0}\right)=$ const,$\quad r_{k}=$ const.

Из (3.9) и стационарности $G$ по $v$ находим искомое вращение $\omega^{\prime 0}, v^{0}$ лишь в виде относительного равновесия $\omega_{0}^{\prime}=0, v^{0}=v_{0}=$ const. Необходимо и достаточно, чтобы параметры $c, v^{0}$ удовлетворяли линейной по $c$ системе уравнений

$$
A c=a, \quad A=\left\|a_{i j}\right\|, \quad a_{i j}=\partial n_{j} / \partial v_{i}^{0},
$$

$\operatorname{det} A \equiv 0, \quad a=1 / 2 \nabla_{v} \Phi\left(v^{0}\right), \quad \Phi \equiv \omega_{0}^{-2} P-n \cdot C n$, 


$$
\begin{gathered}
\mathrm{A}^{3} / 4=\| \begin{array}{l}
2\left[\varrho\left(v_{3}+v_{1} v_{2}\right)-4 v_{1}^{2} v_{3}\right]+\Delta_{1},\left[\varrho\left(\varrho-2 v_{1}^{2}\right)-8 v_{1} v_{2} v_{3}\right]+\Delta_{2}, 4 v_{1}\left(v_{1}^{2}+v_{2}^{2}-v_{3}^{2}-1\right) \\
{\left[\varrho\left(2 v_{2}^{2}-\varrho\right)-8 v_{1} v_{2} v_{3}\right]+\Delta_{3}, \quad 2\left[\varrho\left(v_{3}-v_{1} v_{2}\right)-4 v_{2}^{2} v_{3}\right]+\Delta_{4}, 4 v_{2}\left(v_{1}^{2}+v_{2}^{2}-v_{3}^{2}-1\right)} \\
2\left[\varrho\left(v_{1}+v_{2} v_{3}\right)-4 v_{1} v_{3}^{2}\right]+\Delta_{5}, 2\left[\varrho\left(v_{2}-v_{1} v_{3}\right)-4 v_{2} v_{3}^{2}\right]+\Delta_{6}, \quad 8 v_{3}\left(v_{1}^{2}+v_{2}^{2}\right)
\end{array} \\
\times\left(\varrho=1+\left|v^{2}\right|\right), \quad \Delta_{1}=8 v_{1}^{2}-2 \varrho, \quad \Delta_{3}=2 \varrho-8 v_{2}^{2}, \quad \Delta_{4}=-\Delta_{1}, \\
\Delta_{1}=-8 v_{1} v_{2}, \quad \Delta_{2},
\end{gathered}
$$

адекватной безусловной стационарности $W_{0}$ в $v^{0}$, где $\partial W_{0} / \partial v^{0}=0$. Обозначим

$$
\begin{gathered}
A^{+}=\|A, a\|, \quad r(M)=\operatorname{rank} M, \quad R(\delta)=\left\{v \mid r(A)=r\left(A^{+}\right)=\delta\right\}, \\
R^{+}=\bigcup_{\delta} R^{(\delta)}, R^{\left(\delta_{1}\right)} \cap R^{\left(\delta_{2}\right)}=\varnothing,
\end{gathered}
$$

$\delta_{1} \neq \delta_{2} ; \delta, \delta_{1}, \delta_{2}=\overline{0,2}$. Для существования решения (3.10) необходимо и достаточно $v^{0} \in R^{+}$, где $R^{+}-$допустимое множество $\left\{v^{+}\right\}$. Следует различать $R^{(8)}$. Из $(3.8)$, (3.10) в случаях $v^{0} \in R^{(8)}$ находим, что все решения дают формулы

$c_{0}^{(0)}=\mu_{1} n+\mu_{2} \gamma+\mu_{3} \tau, \quad c_{0}^{(1)}=\mu_{1} n+\mu_{2} g+\alpha_{3}(n \times g), \quad c_{0}^{(2)}=\mu_{1} n+\alpha_{1} \tau+\alpha_{2} \gamma$

$$
(A n=0) \text {, }
$$

где $A g=0, g \cdot n=0,|g|=1, \mu_{i}$ - произвольные, $\alpha_{i}$ - фиксированные постоянные. Из (3.10), (3.11) для (3.3) (например, при гравитирующих $S_{0}, S_{1}$ ) имеем два следствия. Неравенство $l+a^{\prime \prime} \neq 0-$ одна из причин несовпадения триэдров $o_{1} \gamma \tau n$ и $o_{1} e_{i}^{\prime}$. При этом выбором $c$ невозможно получить произвольную стационарную ориентацию триэдров, так как в общем случае $R^{+} \neq E^{3}$. Параметры скрытого движения $c_{i}$ неоднозначно определены параметрами $v_{j}{ }^{0}$ реального наклона триэдров,

Возьмем из (3.4) любое решенне $c_{0}{ }^{*}=c_{0}\left(v^{0}\right) \in\left\{c_{0}{ }^{(\delta)}\right\}$ в допустимой точке $v^{0}$. По теореме Ляпунова для безусловной устойчивости $v=v^{0}$, $\omega^{\prime}=0$ достаточно, чтобы $W_{0}\left(v, c_{0}{ }^{*}\right)$ имела в $v^{0}$ изолированный минимум. В обыкновенной точке $v_{0}^{0}\left(\left|\partial^{2} W_{0}\left(v_{0}^{0}, c_{0}{ }^{*}\right) / \partial v_{i} \partial v_{j}\right| \neq 0\right)$ грубый критерий такой устойчивости дают условия Сильвестра $D_{i}\left(v_{0}^{0}, c_{0}^{*}\right)>0$, $i, j=\overline{1,3}$. В последнем случае для неустойчивости $v_{0}^{0}, 0$, согласно теореме Томсона, достаточно, чтобы в точке $v_{0}{ }^{0}, c_{0}{ }^{*}$ нечетное число этих неравенств изменило знак. Произвол $\mu_{i}$ можно подчинить условию смены устойчивости.

\section{ЛИ Т Е P A T Y P A}

1. Р у мянцев В. В., Вестн. Моск. ун-та, Матем. Мех., № 4, 38-52 (1957).

2. К ей с И., Изв. АН ЭССР, Фнз. Матем., 24, № 3, 277-284 (1975).

3. К е й с И., Изв. АН ЭССР, Фнз. Матем., 28, № 1, 83-85 (1979).

Институт кибернетики Академии наук Эстонской ССР
Поступила в редакцию 29/XII 1979 
I. KEIS

\section{HAMILTONIAANI JA IMPULSSIDE POOLEST LINEAARSE AGREGEERIMISVARIANDIGA LAGRANGE'i SUSTEEMI DUNAAMIKAST}

On leitud invariandi $G$ olekutingimused, kui $G$ kujutab endast impulsside ja hamiltoniaani lineaarset kombinatsiooni holonoomsete ning mitteholonoomsete süsteemide jaoks. $G$-invariandiga mitteautonoomne kanooniline süsteem on teisendatud Hamiltoni autonoomsesse süsteemi. Esitatakse minimaalse mõju printsiibi modifikatsioon. Agregeerimisvariant on leitud sferoidi mittetsentraalses gravitatsiooniväljas ning orbiidil liikuva süsteemi jaoks.

\section{KEIS}

\section{ON THE DYNAMICS OF LAGRANGE SYSTEM WITH THE AGGREGATING INVARIANT LINEAR IN HAMILTONIAN AND THE IMPULSES}

Under the conditions

$$
\begin{aligned}
& \delta^{0} q \triangleq \varepsilon\left(v+v_{0} q \cdot \in\{\delta q\}, \quad Q \cdot \delta^{0} q=0\left(f \cdot=d f / d t, v=\left(v_{k}\right)^{*}\right),\right. \\
& v \cdot \partial H / \partial q-v_{0} \partial H / \partial t=v_{0}{ }_{0} H+v \cdot p\left(u \cdot v=\sum_{k=1}^{n} u_{k} \cdot v_{k}, k=\overline{1, n}\right)
\end{aligned}
$$

the ideal holonomic and non-holonomic Lagrange systems obtain the generalized energy invariant $G \triangleq v_{0} H+v \cdot p=$ const. In the new variables $x, p^{\prime}$ (1.5) every canonic system with $G$-invariant is decomposed and represented in the form of $2(n-1)$-dimensional canonic system independent of the two equations on $x_{n}, x_{n+1}$. The generalized Maupertuis-Lagrange principle is proposed for this system. Various gyrostatic systems have this type of invariant and all the properties noted above. As an example, the aggregating invariant is obtained for the circular gyrostate motion around the gravitating spheroid. The sufficient stability conditions for relative equilibrium are derived in the essentially new attitude parameters $v_{i}(3.8)$. 